\title{
Labyrinthe
}

$13 \mid 2002$

Numéro 13

\section{Patrick Declerck, Les Naufragés : avec les clochards de Paris, Paris, collection « Terre Humaine », Plon, 2001}

\section{Vincent Denis}

\section{(2) OpenEdition \\ Journals}

Édition électronique

URL : http://journals.openedition.org/labyrinthe/1490

DOI : $10.4000 /$ labyrinthe. 1490

ISSN : 1950-6031

Éditeur

Hermann

\section{Édition imprimée}

Date de publication : 15 novembre 2002

Pagination : 145-150

Référence électronique

Vincent Denis, «Patrick Declerck, Les Naufragés : avec les clochards de Paris, Paris, collection « Terre Humaine », Plon, 2001 », Labyrinthe [En ligne], 13 | 2002, mis en ligne le 24 février 2007, consulté le 10 décembre 2020. URL : http://journals.openedition.org/labyrinthe/1490 ; DOI : https://doi.org/10.4000/ labyrinthe. 1490

Ce document a été généré automatiquement le 10 décembre 2020.

Propriété intellectuelle 


\title{
Patrick Declerck, Les Naufragés : avec les clochards de Paris, Paris, collection «Terre Humaine », Plon, 2001
}

\author{
Vincent Denis
}

1 Alors que se closent les campagnes électorales de 2002, et que s'élaborent dans les officines ministérielles les réformes qu'appelle le vote du 21 avril, il est urgent de lire l'ouvrage courageux, critique (violent, même) de Patrick Declerck. Loin des sentiers battus de «l'insécurité » ou de la « crise urbaine », ce psychanalyste belge apporte une contribution de poids à toute réflexion sur la désocialisation. Au-delà, par le biais qu'il adopte, il nous invite à remettre en cause notre relation à la fois aux victimes de l'exclusion, les clochards, et aux enjeux de l'aide sociale. Enfin, par sa démarche et sa réception, ce livre témoigne des carences du débat démocratique dans notre pays.

2 Ce livre apporte d'abord l'expérience de quinze ans de consultations psychiatriques auprès des clochards parisiens. Prenant d'ailleurs une pose modeste, loin d'être un savant traité, il se compose d'une incandescente première partie, "Routes", qui raconte les itinéraires des clochards, mais aussi de l'auteur. Au terme de ce voyage volontiers célinien s'ouvre une seconde partie, plus sage et plus grave, où l'on trouvera les hypothèses scientifiques argumentées. Dans son propos d'ensemble, le livre offre des vue nouvelles sur les mécanismes de la désocialisation. Declerck s'en prend aux objectivations courantes de la désocialisation (sociologiques et psychiatriques), au traitement inadéquat (et à l'idéologie qui la sous-tend, celle de la " réinsertion », par le travail et la règle) dont elle fait l'objet.

3 L'auteur réfute les présupposés de la sociologie comme de la psychiatrie sur les clochards. En en faisant des produits (passifs) d'un processus tout extérieur d'exclusion, au demeurant assez flou puisqu'il permet d'englober toutes sortes d'individus, la sociologie fait des clochards les victimes d'un processus qui dépasse les individus et qui retombe sur la société tout entière. Declerck ne se détache pas entièrement de l'idée que la société sécrète les clochards, mais il s'oppose à l'idée d'une passivité : au contraire, le clochard se caractérise par une pathologie d'autodestruction 
particulièrement active, rarement consciente. La psychiatrie considère généralement le clochard comme extérieur à son champ d'action. C'est pour elle un problème social. De son côté, la sociologie aussi expulse les clochards en renvoyant leur désocialisation profonde dans le domaine de la psychiatrie. Cela conduit l'auteur à se distancier du terme d'« exclusion », pour lui préférer ici celui de "désocialisation», un peu comme Robert Castel avait élaboré le concept de « désaffiliation » dans Les Métamorphoses de la question sociale, autre contribution essentielle à la réflexion sur ces phénomènes.

4 L'apport de Patrick Declerck, fort de son expérience de clinicien au Cash (Centre d'accueil et de soins hospitaliers) de Nanterre, pendant une quinzaine d'années, est de faire de la clochardisation ou de l'état de clochard le symptôme d'une psychopathologie spécifique. S'intéressant aux dix à quinze mille individus profondément désocialisés qui vivent à Paris, et non pas à l'ensemble de la population des SDF, il n'a relevé aucun cas de "réinsertion ", de re-socialisation durable. Selon lui, la pathologie psychiatrique des clochards s'ancre sur une "souffrance-fond", probablement - mais c'est indémontrable - d'origine intra-utérine : une souffrance si forte qu'elle vide le sujet de lui-même. La clinique des clochards est une litanie d'épanchements métaphoriques et littéraux - logorrhées et diarrhées - et d'actes manqués qui conduisent le sujet à s'autodétruire régulièrement. Le clochard est un adulte qui veut retourner au stade utérin : "Mourir. Dormir. Rêver peut-être ", selon le mot de Hamlet que Declerck se plaît souvent à citer. La clochardisation, l'alcoolisme (quasi général) et les polytoxicomanies (fréquentes) sont autant de barrières qu'élève l'individu dans un but anxiolytique, face à une angoisse et à une souffrance autrement insupportables, qui déboucherait peut-être sur le meurtre ou l'effondrement psychotique.

5 La clochardise est bien, selon Declerck, le symptôme d'une pathologie psychiatrique spécifique, ce qui ne veut pas dire qu'elle n'ait pas des origines sociales : au contraire, Declerck insiste sur le caractère multiétiologique de la clochardise. Mais la présence d'individus venus de toutes les couches de la société, et la possibilité pour des individus confrontés à un choc de se clochardiser en quelques mois, quelques jours, voire quelques heures, révèle pour Declerck l'existence d'une pathologie spécifique dont il s'emploie dans la seconde partie du livre, "Cartes", à cerner les contours, et à formuler des pistes thérapeutiques.

6 Le novice découvrira un antimonde dans les récits de Declerck, les discours des clochards, les dossiers reproduits. Soulignons aussi le choix de l'iconographie, où une scène de beuverie chez Jordaens ou un visage halluciné d'Ensor, entrant en résonance avec le texte, suggèrent au lecteur la cohérence d'une expérience des limites que le langage seul ne peut cerner. Corps atrocement meurtris, mutilations volontaires, sexualité repoussante, saleté absolument a-normale : le livre ne cherche pas à éviter la crudité - ici, l'autre nom de la vérité - de ceux que nous ne voulons pas voir, sans pour autant tomber dans les travers louches du voyeurisme. Le lecteur en sort ébranlé, ennuyé aussi parfois, tout comme Patrick Declerck. Ceux qui douteraient encore de la dureté implacable de la vie des clochards, du suicide quotidien qu'elle représente, de l'horreur et des carences de l'hébergement de secours, de l'immensité de la souffrance, se feront une opinion. Quant à l'absurdité de la question d'être SDF « par choix »...

7 L'autre apport majeur de ce livre réside dans sa posture modeste. Publié dans la célèbre collection " Terre Humaine ", et ce n'est pas un hasard, il est fidèle à la ligne définie par son directeur, l'anthropogéographe Jean Malaurie. Il ne s'appuie pas sur une vision englobante et totalisante des sciences humaines. Il implique son auteur dans l'écriture 
ethnographique. Car le livre de Patrick Declerck est aussi le récit de son propre voyage, de son côtoiement des clochards. Traversé d'intérêt, de haine, de compassion et d'ennui, l'auteur s'ausculte: rien n'est simple, rien n'est donné dans cette relation difficile. Patrick Declerck montre admirablement les pièges du sentimentalisme et de la pitié, tout comme ceux de la colère et de la frustration du soignant face à des soignés qui s'autodétruisent.

8 Par la radicalité de son questionnement et de ses conclusions, mais aussi par l'engagement de l'auteur, ce livre incite à jeter un autre regard sur les clochards et les exclus, tout comme sur notre relation avec eux. Celle-ci semble être un grand impensé de notre époque. Passons sur les idéologies de "droite" qui ne voient dans la clochardisation que le résultat d'une faute, au mieux d'un " choix ». Les idéologies de gauche nous poussent finalement toujours à repousser vers l'État les exclus, et à attendre finalement d'une politique de l'emploi volontariste et énergique une éradication ou au moins une amélioration sensible du sort de ces malheureux. Depuis dix ans, le phénomène ne semble pas avoir cessé de s'accroître, dans l'indifférence générale, comme si nous refusions tous de voir en face la mort (pas si lente, et pas très douce) d'autres gens en bas de nos immeubles, toute l'année. Pourquoi ce silence, en dehors de quelques cris périodiques au moment des grands froids?

Ce que montre très bien Declerck, c'est l'inadéquation du «traitement» de la désocialisation, et de l'idéologie qui le sous-tend. Poids d'une tradition carcérale qui remonte au moins à l'Hôpital général ou peut-être même au bas Moyen Âge selon Robert Castel, faite d'ambivalence vis-à-vis du pauvre : la punition et l'assistance, la souffrance et le redressement, le sadisme et la charité se mélangent toujours, dans une incroyable continuité, à travers le traitement des clochards. Le clochard - comme la prostituée, le toxicomane, le délinquant ou l'homosexuel - est une figure de la transgression. C'est pourquoi il importe toujours qu'il souffre même dans les lieux censés le protéger et l'assister. La société doit toujours montrer à ceux qui souhaiteraient se détacher de la normalité que "l'alternative » (comme s'il s'agissait d'un choix, ici) est hasardeuse, cruelle et douloureuse. L'incurie administrative vient en renfort de cette petite musique continue de souffrances (pas trop méchantes, car sinon elles produiraient l'inverse de l'effet désiré) pour élargir l'écart entre les grands discours et les réalités. Il faut lire les descriptions hallucinantes des nuits au foyer de Nanterre, et l'évolution ambiguë du Cash ces dernières années : à vrai dire, avec les conditions pour qu'une insécurité croissante y règne, et le détournement dont la structure fait l'objet, par des populations particulièrement inquiétantes, le tableau est apocalyptique, et l'on n'est pas prêt de voir «zéro SDF » dans les rues, bien au contraire.

10 C'est une leçon à méditer pour les réformes judiciaires et sociales qui se préparent, et sur lesquelles les citoyens ont été appelés à se prononcer ou vont l'être. Au passage, je souligne l'incomparable richesse du témoignage de Patrick Declerck, qui met ainsi à la portée de tous le fruit de son expérience, au lieu de la laisser à un quelconque rapport administratif qui finira aux archives (pour le bonheur exclusif des historiens futurs). En tant que citoyens, nous avons été invités à nous prononcer sur des programmes tout entiers centrés sur l'insécurité. Or, le travail honnête d'une partie de la presse ${ }^{1}$ ne peut suppléer à un ouvrage de la qualité de celui de Patrick Declerck. L'espace éditorial y est trop étroit, et il y règne une tendance à croire que l'on domine le réel par la statistique et les enquêtes de l'Insee ou de l'Ined, dont les résultats publiés se limitent en fait à des 
«photographies» de la société, de ses "pathologies», et qui n'apportent aucun éclairage sur le fonctionnement des institutions, alors que c'est sur cela que le débat doit porter. Il est dommage qu'au moment où a été proférée la fameuse formule « zéro SDF » aucun grand média n'ait sollicité les acteurs de la lutte contre la désocialisation (Patrick Declerck a seulement publié un court texte dans Le Monde sur le mode du " chiche »). La grande question était de savoir si c'était une idée de Jacques Seguela ou du candidat... Trêve d'ironie : les réformes judiciaires et les nouveaux traitements de la délinquance se décideront probablement sans nous, citoyens, faute d'une information suffisante, et d'un accès à l'espace public problématique pour les acteurs de ces réformes (magistrats, policiers, travailleurs sociaux) : le devoir de réserve existe pour tous ces agents de l'État, mais que pouvons-nous savoir, autrement? Ce n'est pas aux seuls sociologues d'évaluer de l'extérieur l'efficacité et la pertinence des politiques publiques : il y a un savoir spécifique des acteurs qui ne peut être restitué que par eux².

11 Patrick Declerck remet en question les relations qui s'instaurent entre clochards et agents sociaux, entre soignants et soignés. Les clochards payent en monnaie symbolique l'assistance, en montrant leur bonne volonté de réinsertion, mot fétiche et au coeur de l'idéologie du travail qui structure le traitement de la désocialisation. Se produisent alors un transfert et un contre-transfert (du soignant sur le soigné) qui est préjudiciable à la relation. Le clochard est sommé de se réinsérer, de se normaliser, alors qu'il en est justement incapable. Pendant le traitement thérapeutique, il doit par magie « suspendre » toutes ses pratiques déviantes, punies par l'exclusion et la fin de la thérapie. Declerck montre comment ces pratiques sont anxiolytiques et procurent du plaisir, des bénéfices au clochard: il est d'autant plus difficile de les quitter (" l'abstinence, c'est beau, mais c'est triste », disait un de ses patients). En même temps, le clochard est confronté à l'angoisse d'être réinséré dans le monde « réel » avec toutes les contraintes et les angoisses qu'il est incapable d'affronter. Se noue alors une spirale sans fin de chutes, d'actes manqués et de reprises de la thérapie dans une nouvelle institution, suivies de nouveaux échecs, jusqu'à la déchéance définitive et la mort du clochard. Patrick Declerck invite à repenser les notions inadéquates d'échec et de succès thérapeutiques, car ici en l'occurrence la thérapie se trompe de but: il faut accepter la chronicité du mal, son irréversibilité. Il faut accepter d'aider des hommes et des femmes tels qu'ils sont, de les empêcher de se faire trop mal quand ils tombent, de leur procurer aussi du plaisir. Aujourd'hui, écrit Patrick Declerck, il n'y a plus d'espace où l'on peut être fou ${ }^{3}$. Sommes-nous prêts à les accepter et à nous occuper d'eux, tels qu'ils sont, sans vouloir les punir, sans vouloir à tout prix les engager dans une normalisation condamnée d'avance? La question vaut aussi pour d'autres catégories : sommes-nous prêts à accepter les laissés-pour-compte ? Le débat sur la question sociale ne doit pas être confisqué par l'extrême-gauche, avec pour seul horizon une utopique redistribution des richesses qui supprimerait ces souffrances. La question est autrement plus urgente, et d'ailleurs l'extrême gauche de tradition marxiste ne voit dans le vagabond qu'un parasite, au mieux, ou un traitre dont la bourgeoisie achète le silence et la "dignité » par la charité. On voit quelles questions entraîne peu à peu le livre, au-delà de ce groupe de quelques milliers de clochards parisiens. 


\section{NOTES}

1. La tendance à proposer au lecteur des « données " prétendument «brutes » (notamment sous la forme de graphiques) pour l'inciter à réfléchir et à se faire son propre jugement, fort louable, peut produire l'effet opposé : cette présentation naturalise des phénomènes construits et conduit à les réifier. De même, en simplifiant souvent des études et des enquêtes en quelques données statistiques, elle risque de conduire chacun à retrouver des idées simples (comme " la délinquance augmente », ou " les étrangers sont plus nombreux à être au chômage que les Français »). La réaction devant ces articles qui abordent rarement la question pourtant essentielle de ce qu'ils présentent comme des « problèmes » ou des « dossiers » (donc des pathologies) va du découragement à l'exaspération en passant par l'inquiétude. Tout est abordé selon l'angle de la recherche des « causes » (or, il n'y a pas de causalité linéaire simple en sciences humaines), rapidement citées, la description des problèmes, et enfin le « y-aqu'à » incantatoire de quelque spécialiste (député auteur d'un rapport, ou chercheur) où l'analyse du traitement est réduit à la portion congrue.

2. La parution de le livre de Véronique Vasseur sur son expérience de médecin-chef à la Santé en 1999 a ouvert un débat salutaire sur la prison, et l'ampleur du phénomène qui s'en est ensuivi (de l'édition à la télévision) a montré qu'un espace et une demande pour ces questions peuvent exister. Ce livre cru et peut-être discutable a posé les bases d'un débat public renouvelé sur le pénal, mieux que la sociologie pénitentiaire depuis vingt ans. Non pas que celle-ci soit inopérante : au contraire, elle est cruciale. Mais elle ne peut prétendre épuiser l'ensemble du débat et elle doit probablement y participer avec les acteurs, sans exclusion. Enfin, il faut reconnaître que les ouvrages des sociologues n'ont pas toujours frayé la voie à des débats publics - même si quelques exceptions brillantes, comme La Misère du monde ou Les Métamorphoses de la question sociale, viennent s'opposer à la règle qui veut que l'ouvrage « grand public » et le « témoignage » suscitent des débats plus larges, comme pour la prison ou les clochards. 3. À juste titre, Patrick Declerck montre que la volonté normalisatrice se niche de la manière la plus improbable dans les projets les plus généreux. L'hymne des Restos du cœur ne commence-t-il pas ainsi ?: « Aujourd'hui, on n'a plus le droit d'avoir faim ni d'avoir froid.» 